\title{
Cerebral monitoring during carotid endarterectomy using transcranial cerebral oximetry
}

\author{
Sihotsky $\mathrm{V}^{1}$, Berek $\mathrm{P}^{1}$, Kopolovets $\mathrm{I}^{1}$, Frankovicova $\mathrm{M}^{1}$, Stefanic $\mathrm{P}^{1}$, Kubikova $\mathrm{M}^{1}$, Mucha $\mathrm{R}^{2}$ \\ Department of Vascular Surgery, Eastern Slovak Institute of Cardiovascular Diseases and Faculty of Medicine, \\ PJ Safarik University, Kosice, Slovakia. berekp67@gmail.com
}

\section{ABSTRACT}

OBJECTIVE: This study aimed to evaluate the outcomes of carotid endarterectomies (CEA) performed using transcranial cerebral oximetry as cerebral monitoring

METHODS: This single-center study included patients treated surgically for significant stenosis of the internal carotid artery (ICA) from January 2012 to December 2017

RESULTS: Of the 654 patients, 267 were asymptomatic, and 387 were symptomatic. Eversion CEA was performed in $64.8 \%$, and patched conventional CEA in $35.2 \%$ of all patients. Totally $11.4 \%$ of all patients had a shunt inserted; all patients with the inserted shunt had the conventional pathed CEA. Severe postoperative complications occurred in $1.5 \%$ of asymptomatic patients and $2.6 \%$ of symptomatic patients. The stroke and death ratio in the shunted group was $2.7 \%$, and in the non-shunted group, $2.1 \%(p=$ 0.7 ). We found no significant difference in severe postoperative complications between the shunted and non-shunted group. Further, we found the male gender $(p=0.005)$, coronary artery disease $(p=0.01)$, and ongoing smoking $(p=0.003)$ to be significantly associated with neurological symptoms of the ICA stenosis. We also confirmed current tobacco smoking to be significantly associated with the occurrence of postoperative stroke and death $(p=0.005)$

CONCLUSION: We found transcranial cerebral oximetry to be reliable in the determination of shunt insertion (Tab. 6, Ref. 26). Text in PDF www.elis.sk.

KEY WORDS: carotid endarterectomy, transcranial cerebral oximetry.

\begin{abstract}
Abbreviations: CAD - coronary artery disease, CEA - carotid endarterectomy, ICA - internal carotid artery, MI - myocardial infarction, TCD - transcranial Doppler, TCO - transcranial cerebral oximetry, TIA - transient ischemic attack
\end{abstract}

\section{Introduction}

Stroke is the second most common cause of death in developed countries (1). More than half of stroke survivors stay dependent on others for some aspect of everyday activities (2). Thus, stroke is the most frequent reason for an older population's disability. Stroke also imposes an enormous financial burden on the health system (3). Carotid artery stenosis is a well-established cause of brain ischemia, accounting for up to $20 \%$ of stroke cases $(4,5)$. Carotid endarterectomy (CEA) represents one of the most efficient preventions of stroke. Recently, carotid endarterectomy has been

${ }^{1}$ Department of Vascular Surgery, Eastern Slovak Institute of Cardiovascular Diseases and Faculty of Medicine, Pavol Jozef Safarik University, Kosice, Slovakia, and ${ }^{2}$ Institute of Neurobiology, Biomedical Research Center of Slovak Academy of Sciences, Kosice, Slovakia

Address for correspondence: $\mathrm{P}$. Berek, MD, PhD, Department of Vascular Surgery, Eastern Slovak Institute of Cardiovascular Diseases and Faculty of Medicine, Pavol Jozef Safarik University, Ondavska 8, SK-040 01 Kosice, Slovakia.

Phone: +421.55.7891510, Fax: +421.55.7891513 indicated for asymptomatic patients with more than $70 \%$ stenosis of the internal carotid (ICA) artery and for symptomatic patients with more than $50 \%$ stenosis of the internal carotid artery (3).

During a carotid endarterectomy, the internal carotid artery must be clamped, but in the majority of patients, the collateral brain circulation is sufficient, and they tolerate the carotid crossclamping well. Only about $15 \%$ of the patients have an insufficient collateral system, and they require inserting a shunt (6). The shunt is inserted into the common and internal carotid artery and enables blood supply from the common into the internal carotid artery and bridges the clamped carotid territory. But the insertion of the shunt itself can also cause a stroke by dissection of the arterial wall or by air embolism. Thus, selective placement of a shunt, selective shunting, is the preferred method of shunting among vascular surgeons $(7,8)$. In selective shunting, the shunt is placed only in the case when the blood supply to the brain after carotid cross-clamping is not sufficient. Carotid endarterectomy is performed mainly in general anesthesia, so the operating team needs a technique that can determine impaired brain circulation after carotid cross-clamping and which indicates a shunt insertion (9). Standard methods for determination of a shunt insertion are transcranial doppler (TCD) or somatosensory evoked potentials $(5,10)$.

TCD needs a temporal bone window in the skull, and about $15 \%$ of patients do not have it (11). The other disadvantage of the transcranial doppler is that it requires the presence of another 
431-436

physician in the operating theatre. Somatosensory evoked potentials are not precise and are modified by general anesthesia (7). Transcranial cerebral oximetry (TCO), also termed near-infrared spectroscopy, seems to be a novel method of brain circulation monitoring during carotid endarterectomy. This method allows for continuous monitoring of cerebral hemoglobin oxygen saturation in frontal lobes using near-infrared light (12). TCO is easy to interpret and appears to be reliable in the determination of shunt insertion $(9,10,11,13)$.

\section{Patients and methods}

\section{Patients}

The single-center study was performed at the Department of Vascular Surgery, East Slovak Institute of Cardiovascular Diseases and Faculty of Medicine, University of Pavol Jozef Safarik, Kosice. Six hundred fifty-four patients with significant stenosis of the internal carotid artery, who were referred to our department from January 2012 to December 2017, were involved in the study. Symptomatic patients were defined as patients who had suffered transient ischemic attack (TIA) or ischemic stroke within the last 180 days, while asymptomatic patients were defined as those who did not suffer any TIA or stroke within the previous 180 days. Significant stenosis of the internal carotid artery was defined as stenosis of more than $50 \%$ of the lumen in symptomatic patients and more than $70 \%$ in asymptomatic patients. The degree of the ICA stenosis was determined using digital subtraction angiography. Contraindications for the operation (exclusion criteria) were: chronic ICA occlusion, restenosis after carotid endarterectomy, previous neck operation, previous neck radiotherapy, tracheostomy, palsy of the recurrent laryngeal nerve, unstable angina pectoris, recent heart failure, recent myocardial infarction (MI), recent cardiac surgery, the ejection fraction under $30 \%$, acute infection. Informed consent was obtained from all participants in the study. All patients who met the criteria for carotid endarterectomy but were high-risk for surgery were reffered for carotid stenting.

\section{Procedure and postoperative course}

All patients were operated in general anesthesia. We were selective shunters, and a shunt was inserted only in the case of reduced blood supply to the brain. We used transcranial cerebral oximetry as cerebral monitoring during general anesthesia. The device INVOSTM 5100 C, Somanetics, Troy, MI, U.S.A., was used for measurement of cerebral saturation. In all patients the initial value of brain saturation after introduction into anesthesia, before carotid cross-clamping was assessed. The second value of cerebral saturation was measured one minute after carotid-cross clamping. When the decrease of the cerebral saturation after clamping was more than $20 \%$, the Brenner shunt was inserted into the common and internal carotid artery. Eversion or conventional carotid endarterectomy with a patch were performed baccording to the surgeon's decision. We preferred the eversion carotid endarterectomy. Conventional endarterectomy with a patch was done mainly in the cases when the shunt insertion was necessary or when the atherosclerotic process at the ICA was advanced and proceeded more distally. All patients were prescribed with antiplatelet therapy postoperatively and were evaluated by an independent neurologist after the operation. Postoperative stroke, myocardial infarction, and death were considered as major, severe complications and cranial nerve injury or postoperative hematoma as minor complications.

\section{Statistical analysis}

Results were evaluated using software GraphPad Prism 5.0 (GraphPad Software Inc., La Jolla, CA), we applied chi-square test. Statistical significance was set according to the $p$ value. $p$ value $0.05-0.01=*, 0.01-0.001=* *,<0.001=* * *$.

\section{Results}

A total of 654 (412 men and 242 women) were included in the study. The mean age of patients was $70.1 \pm 6.1$ years. Two hundred sixty-seven patients (40.8\%) had asymptomatic ICA stenosis, and three hundred eighty-seven patients (59.2\%) had symptomatic ICA stenosis. Diabetes had $27.1 \%$, hypertension $93.0 \%$, coronary artery disease (CAD), $46.9 \%$, and chronic pulmonary disease, $18.1 \%$ of patients. Current smokers were $15.0 \%$, and contralateral carotid artery occlusion had $3.1 \%$ of patients. The basic characteristics of the patients with underlying diseases are summarized in Table 1.

We used selective shunting, and the shunt was inserted into the ICA when the decrease of transcranial cerebral saturation after one

Tab. 2. Major and minor postoperative complications in asymptomatic and symptomatic patients.

\begin{tabular}{lcclcc}
\hline \multirow{2}{*}{ Complications } & \multicolumn{2}{c}{ Asymptomatic } & & \multicolumn{2}{c}{ Symptomatic } \\
\cline { 2 - 3 } \cline { 5 - 6 } & No (267) & $\%$ & & No (387) & $\%$ \\
\hline Stroke & 4 & 1.5 & & 7 & 1.8 \\
Death & 0 & 0.0 & & 3 & 0.8 \\
Myocardial infarction & 1 & 0.4 & & 2 & 0.5 \\
Stroke + death & 4 & 1.5 & & 10 & 2.6 \\
Stroke + death +MI & 5 & 1.9 & & 12 & 3.1 \\
Bleeding & 8 & 3.0 & & 9 & 2.3 \\
Lesion of cranial nerves & 7 & 2.6 & & 12 & 3.1 \\
\hline
\end{tabular}


Tab. 3. Stroke and death ratio according to shunt insertion.

\begin{tabular}{lccccc}
\hline & with shunt & without shunt & all & p & significance \\
\hline No patients & 75 & 579 & 654 & & \\
stroke+death (No) & 2 & 12 & 14 & & \\
stroke+death (\%) & $2.70 \%$ & $2.10 \%$ & $2.10 \%$ & 0.7 & N/S \\
\hline
\end{tabular}

Tab. 4. Stroke and death ratio according to the type of endarterectomy.

\begin{tabular}{lccccc}
\hline & Conventional CEA & Eversion CEA & All & $\mathrm{p}$ & significance \\
\hline No patients & 230 & 424 & 654 & & \\
stroke + death (No) & 6 & 8 & 14 & & \\
stroke + death (\%) & $2.6 \%$ & $1.9 \%$ & $2.10 \%$ & 0.5 & $\mathrm{~N} / \mathrm{S}$ \\
\hline
\end{tabular}

$\mathrm{NS}$ - non-significant

Tab. 5. Association between carotid artery symptoms and gender and underlying diseases.

\begin{tabular}{lcccc}
\hline & Asymptomatic & Symptomatic & $\mathrm{p}$ & Significance \\
\hline No & 267 & 387 & & \\
Men & 133 & 279 & 0.005 & $* *$ \\
Women & 134 & 108 & 0.0001 & $* * *$ \\
Diabetes & 67 & 110 & 0.4 & $\mathrm{~N} / \mathrm{S}$ \\
Hypertension & 238 & 370 & 0.5 & $\mathrm{~N} / \mathrm{S}$ \\
CAD & 101 & 206 & 0.01 & $*$ \\
Pulmonary disease & 42 & 76 & 0.3 & $\mathrm{~N} / \mathrm{S}$ \\
Current smoker & 25 & 73 & 0.003 & $* *$ \\
Contralteral occlusion & 6 & 14 & 0.3 & $\mathrm{~N} / \mathrm{S}$ \\
\hline
\end{tabular}

CAD - coronary artery disease. $*$ p $0.05-0.01, * * 0.01-0.001, * * *<0.001$

minute of carotid cross-clamping was $20 \%$ or more. We inserted shunt in totally 75 patients $(11.4 \%)$. All patients with implanted shunt had patched conventional carotid endarterectomy. Eversion carotid endarterectomy was performed in 424 cases (64.8\%). Eversion carotid endarterectomy was all the time without a shunt. Patched conventional carotid endarterectomy was performed in 230 patients $(35.2 \%)$, and in 75 patients using a shunt (11.4\%) and 155 patients had patched conventional carotid endarterectomy without a shunt $(23.7 \%)$.

Severe postoperative complications (stroke, death, myocardial infarction) occurred in five asymptomatic patients $(1.9 \%)$. Four patients $(1.5 \%)$ had a stroke, and one patient $(0.4 \%)$ had a myocardial infarction. Minor complications were observed in fifteen cases $(5.6 \%)$. Totally seven patients $(2.6 \%)$ had a postoperative transient lesion of cranial nerve and eight patients (3.0 $\%$ ) had postoperative bleeding requiring surgical revision (Tab. 2 ). In symptomatic patients, severe postoperative complications occurred in twelve patients $(3.1 \%)$. Seven patients $(1.8 \%)$ suffered a postoperative stroke, three patients $(0.8 \%)$ died, and two patients $(0.5 \%)$ had a myocardial infarction. Minor postoperative complications were observed in twenty-one patients (5.4\%) in the cohort of symptomatic patients. Twelve patients $(3.1 \%)$ had a postoperative lesion of cranial nerves, and nine patients $(2.3 \%)$ had surgical revision due to postoperative bleeding (Tab. 2).

We assessed the occurrence of severe postoperative complications as stroke and death according to shunt placement. In the group of patients with inserted shunt, severe complications occurred in two cases $(2.7 \%)$. In the group without shunt inserted during carotid endarterectomy, severe complications occurred in twelve cases $(2.1 \%)$. The difference was not statistically significant $(\mathrm{p}=0.7)$ (Tab. 3).

The occurrence of postoperative stroke and death was also assessed according to the type of endarterectomy. Patients with eversion carotid endarterectomy had severe complications in eight cases (1.9\%), and patients with conventional patched endarterectomy had a severe complication in six cases $(2.6 \%)$. The difference between both groups was not significant $(\mathrm{p}=0.5)$ in Table 4.

We also compared patients with symptomatic stenosis of the internal carotid artery stenosis with asymptomatic carotid artery stenosis according to gender and underlying disease using the chiquadrate test. We found that symptomatic stenosis of the ICA was significantly associated with male gender $(\mathrm{p}=0.005)$, with CAD $(p=0.01)$ and current smoking $(p=0.003)$ ( Table 5.). Other underlying diseases were not significantly associated with neurological symptoms of ICA.

We also analyzed postoperative complications and the occurrence of underlying diseases. We found that patients with postoperative stroke and deaths (14 patients) were smokers in eight cases $(57.1 \%)$ compared to $14.9 \%$ of current smokers in the whole group. The difference was significant with $\mathrm{p}=0.005$. Pa-

Tab. 6. Characteristics of the patients with postoperative complications.

\begin{tabular}{|c|c|c|c|c|c|}
\hline & All patients & $\begin{array}{c}\text { Patients with complications } \\
\text { (stroke+death) }\end{array}$ & $\mathrm{p}$ & $\begin{array}{c}\text { Patients with complications } \\
\text { (stroke+death }+\mathrm{MI})\end{array}$ & $\mathrm{p}$ \\
\hline Total & 654 & 14 & - & 17 & - \\
\hline Men & $412(63.0 \%)$ & $10(71.4 \%)$ & 0.929 & $12(70.5 \%)$ & 0.916 \\
\hline Women & $242(37.0 \%)$ & $4(28.6 \%)$ & 0,854 & $5(29.5 \%)$ & 0.838 \\
\hline Diabetes & $177(27.1 \%)$ & $7(50.0 \%)$ & 0.291 & $8(47.1 \%)$ & 0.301 \\
\hline Hypertension & $608(92.2 \%)$ & $13(92.8 \%)$ & 0.847 & $15(88.2 \%)$ & 0.975 \\
\hline CAD & $307(46.9 \%)$ & $13(92.8 \%)$ & 0.117 & $16(94.1 \%)$ & 0.071 \\
\hline Current smoker & $98(14.9 \%)$ & $8(57.1 \%)$ & $0.005 * *$ & $9(58.8 \%)$ & $0.004 * *$ \\
\hline
\end{tabular}

MI - myocardial infartion. $\mathrm{p}^{*} 0.05-0.01, * * 0.01-0.001, * * *<0.001$ 
tients with postoperative stroke, death, and myocardial infarction (17 patients) were smokers in nine cases (58.8\%) compared with $14.9 \%$ of currents smokers in the whole group. The difference was also significant with $\mathrm{p}=0.004$. CAD was also more frequent in the group of patients with postoperative stroke, death, and myocardial infarction, sixteen cases $(94.1 \%)$ compared to the 46.9 $\%$ of patients with CAD in the whole cohort. The difference was marginally significant with $\mathrm{p}=0.07$ (Tab. 6).

A comparison of severe postoperative complications in the group of patients with the symptomatic ICA stenosis and asymptomatic ICA stenosis was performed. The difference in stroke and death ratio between the symptomatic $(2.6 \%)$ and asymptomatic patients $(1.5 \%)$ was not significant $(\mathrm{p}=0.516)$. Also, the difference in the postoperative occurrence of stroke, death, and MI in the group of symptomatic patients $(3.1 \%)$ and asymptomatic patients $(1.9 \%)$ was not significant $(\mathrm{p}=0.486)$.

\section{Discussion}

Carotid endarterectomy is an efficient prevention of stroke in patients with severe stenosis of the internal carotid artery and can be performed in regional or general anesthesia. About $85 \%$ of patients with the ICA stenosis have sufficient collateral system, and blood flow is not reduced by carotid cross-clamping (6). The rest of the patients needs the insertion of a shunt to maintain cerebral circulation during operation. There are two acceptable possibilities of shunting: routine and selective. By routine shunting, a shunt is inserted in all cases. The problem is that about $85 \%$ of shunts are placed unnecessarily. The insertion of the shunt can cause the stroke itself, mainly by dissecting the distal intima at the ICA or by embolization during shunt insertion. Selective shunting avoids unnecessary shunt insertion and seems to be more feasible than routine shunting (7). But the operating team needs a method for determining brain ischemia during shunting.

The best monitoring of neurological functions during carotid cross-clamping seems to be the observation of the patient's consciousness during regional anesthesia $(6,14)$. But general anesthesia brings more comfort for the patients and also for the operating team that is not disturbed by the patient's movement during surgery. Studies, as the GALA trial, have compared general and regional anesthesia, and they did not find any difference in the occurrence of severe postoperative complications in patients operated in general and regional anesthesia $(15,16)$. Also, an extensive metaanalysis of fourteen studies found the rate of severe postoperative complications in patients operated in regional anesthesia to be 3.2 $\%$ and $3.5 \%$ in patients operated in general anesthesia. The difference was not significant. Based on the outcomes of the GALA trial and also this meta-analysis $(15,16)$, international societies consider both general and regional anesthesia to be equivalent (3, 14). The majority of surgeons prefer general anesthesia during carotid endarterectomy. Hence, more methods to establish brain ischemia during CEA in general anesthesia were advanced. Standard techniques of cerebral monitoring during general anesthesia are transcranial doppler (TCD), evoked somatosensory potential, and stumpf pressure measurement.
But those methods also have disadvantages. TCD is precise but needs a temporal bone window for assessing intracranial blood flow and is absent in about $15 \%$ of patients (17). Other disadvantage of TCD is the necessity of another physician in the operating theatre to interpret TCD. Somatosensory evoked potentials can be modified by general anesthesia and are not so precise (18). Measurement of the stumpf pressure is an obsolete technique.

Transcranial cerebral oximetry represents a novel method of cerebral monitoring during carotid endarterectomy. TCO non-invasively detects saturation of brain tissue using transilluminating spectroscopy. Electrodes are placed on the patients forehead and allow continuous monitoring of cerebral hemoglobin oxygen saturation in frontal lobes using near-infrared light (19). This method started to be used in cardiac surgery in the 1980s (12). TCO is an easy to interpret technique, and does not require the presence of another physician at the operating theatre. The operating surgeon himself can interpret TCO.

Samra et al. published the first study evaluating TCO during carotid endarterectomy in 2000 (20). He compared TCO and awake test in patients operated in the cervical block. In 99 patients, he found that TCO had only $2.6 \%$ false-negative results. It means that TCO was not able to detect the patient that needed the shunt insertion only in $2.6 \%$. The authors used a $20 \%$ decrease in cerebral saturation after carotid clamping as cut- off value for shunt placement.

Similarly, Ali et al in 2011 compared TCO, TCD, and the awake test in 49 patients operated in regional anesthesia, and the drop in TCO of $20 \%$ or more was used as a cut-off for shunt placement. They found that TCO had $2.0 \%$ of false-negative and $6.0 \%$ of false-positive results (11). Even better results were published by Ritter et al in 2011. The authors also compared TCO and the awake test in 83 patients that were operated in the cervical block and found $0.0 \%$ false-negative, and only $2.6 \%$ of false-positive results (10). The technique was able to detect all patients who needed the shunt according to the awake test. They used a $19 \%$ decrease of TCO after clamping as a criterion for the shunt insertion. Pedrini et al compared TCO and the stumpf pressure in a more extensive cohort of 473 patients that were operated in general anesthesia and found $0.0 \%$ of false-negative and only $4.6 \%$ of false-positive results (9). Pedrini et al. also used a $20 \%$ decrease as a cut-off value for the shunt insertion; they reported no postoperative stroke or death. Wang with coworkers in 2019, compared TCD and TCO in 84 patients undergoing carotid endarterectomy in general anesthesia, the false-positive results and positive were worse, but they used $12.5 \%$ decrease as a cut-off value for inserting (21). However, the authors reported no postoperative stroke or death in their cohort. Recently, Jonnson et al, in a group of 184 patients operated in cervical block, found TCO to have excellent specificity and acceptable sensitivity in predicting brain ischemia during carotid endarterectomy (13). Only $2.7 \%$ of the patients suffered stroke postoperatively, and postoperative MI occurred in $1.6 \%$. Based on the mentioned studies, we can assume that TCO has almost 0.0 $\%$ of false negativity. That means that TCO can determine nearly all patients that require shunt according to other methods of neuromonitoring. Transcranial cerebral oximetry has false positivity from 2.6 to $4.6 \%$, so up to $4.6 \%$ indicates the shunt placement 
unnecessarily $(9,10,11,13)$. Studies using TCO reported the occurrence of postoperative stroke and death by up to $2.7 \%$ (13).

At our department, we used TCO as neuromonitoring during carotid endarterectomy, and we also applied a $20 \%$ decrease of TCO after carotid clamping as a criterion for shunt insertion. To our knowledge, our group of 654 patients is the most extent published study concerning transcranial cerebral oximetry during carotid endarterectomy. We did not compare TCO with the other method of neuromonitoring, but statistical evaluation showed that patients with inserted shunt had postoperative stroke and death in $2.7 \%$, and in the group of patients without an inserted shunt, the occurrence of postoperative stroke and mortality was $2.1 \%$. The difference was not significant $(\mathrm{p}=0.7)$. Both groups of patients, with the shunt and without the shunt, had the same rate of severe postoperative complications. These results confirm that TCO is secure in detecting patients requiring shunt placement. If TCO did not indicate the shunt placement reliable, the group of patients without shunt would have more severe postoperative complications due to unrecognized brain ischemia during carotid cross-clamping. Furthermore, the occurrence of postoperative stroke and death in asymptomatic patients, $1.5 \%$, and $2.6 \%$ in symptomatic patients confirm the safety of TCO.

There are two possibilities of carotid endarterectomy: conventional and eversion. Eversion endarterectomy is quicker compared to the conventional, but it is almost impossible to implant a shunt during this procedure. Conventional is a more feasible option in case of shunt insertion or when the atherosclerotic process proceeds further from carotid division. Conventional endarterectomy should not be performed with direct suture, but only using a patch. The first study comparing eversion and conventional endarterectomy was the EVEREST study that found eversion endarterectomy to be associated with significantly lesser restenosis compared to the conventional endarterectomy, but those restenoses did not lead to more neurological complication (22). A meta-analysis of eleven randomized and non-randomized trials showed the rate of severe postoperative complications of eversion and conventional patched endarterectomy without a significant difference (23). Also, recommendations of international societies admit usage of both types of carotid endarterectomy. But classic endarterectomy should be used with a patch $(3,14)$.

In our cohort was the postoperative stroke and death rate in the group of patients with eversion endarterectomy $1.9 \%$ while in the group of patched endarterectomy, it was $2.6 \%$ - the difference between that two groups was not significant. We, at our department, prefer eversion endarterectomy, we have performed eversion endarterectomy in $64.8 \%$ of all cases in our study. We reserve conventional only for patients when shunt must be inserted or when longer part of the ICA is involved in the atherosclerotic process, then more advanced atherosclerotic lesion can be better treated by conventional endarterectomy.

We confirmed that patients undergoing carotid endarterectomy were polymorbid. Diabetes had $27.1 \%$, hypertension $93.1 \%$, CAD $46.9 \%$, pulmonary disease $18.1 \%$ and contralateral carotid occlusion $3.1 \%$ of all patients. Current smoking was present in 15.0 $\%$ of patients. We also performed a statistical analysis between asymptomatic and symptomatic patients regarding gender and underlying diseases. We found the male gender $(p=0.005), C A D$ $(p=0.01)$, and smoking $(p=0.003)$ to be significantly associated with symptomatic ICA stenosis. These results are in line with the finding of previous studies that the male gender is a risk factor for stroke (24). Current smokers have a four-time higher risk of stroke compared to non-smokers (4). We did not confirm other well-established risk factors for stroke as hypertension or diabetes (3), probably because of the small number of patients in our study.

The ECST and NASCET trial found female gender, hypertension, contralateral carotid occlusion, and the presence of preoperative neurological symptoms to be associated with significantly more postoperative complications (3). Bennet with coworkers confirmed contralateral carotid occlusion and presence of neurological symptoms before operations as a risk factor for postoperative complications $(25,26)$ and even found other risk factors for postoperative complications as ongoing tobacco smoking, CAD, and congestive heart failure. In our analysis of 17 patients that suffered severe postoperative complications, we confirmed current tobacco smoking to be significantly associated with postoperative stroke and death $(\mathrm{p}=0.005)$, and also with postoperative stroke, death, and myocardial infarction $(\mathrm{p}=0.004)$. We also found CAD to be marginally associated with the postoperative occurrence of stroke, death, and myocardial infarction $(\mathrm{p}=0.07)$. We did not confirm significance for other risk factors as female gender, preoperative neurological symptoms, contralateral occlusion, or hypertension, probably because of the small number of the group of patients with severe postoperative complications.

\section{Conclusion}

Carotid endarterectomy represents a prevention of stroke in patients with internal carotid artery stenosis and is indicated in patients with asymptomatic stenosis over $70 \%$ and in patients with symptomatic stenosis over $70 \%$. The rate of severe postoperative complications should not exceed $3 \%$ in asymptomatic patients and $6 \%$ in symptomatic patients. We perform carotid endarterectomy in general anesthesia, and we use transcranial cerebral oximetry as neuromonitoring during the surgical procedure. In our study, we confirmed that the severe complications rate in patients with inserted shunt was not significantly different from the severe complications rate in patients without the shunt. Thus, we found transcranial cerebral oximetry to be reliable in the determination of shunt insertion. We have also confirmed that the severe complications rate in patients after eversion CEA was not significantly different from those after conventional patched CEA. We found male gender, $\mathrm{CAD}$, and ongoing tobacco smoking to be significantly associated with neurological symptoms of the ICA stenosis. Statistical analysis showed current smoking to be significantly associated and also CAD to be marginally significantly associated with the occurrence of postoperative complications. The presence of severe postoperative complications in $1.5 \%$ of asymptomatic patients and $2.6 \%$ of symptomatic patients indicates the reliability of transcranial cerebral oximetry in detecting cerebral ischemia during operation. 
$431-436$

\section{References}

1. Nichols M, Townsend N, Luengo-Fernandez R, et al. (2012). European Cardiovascular Disease Statistics 2012. European Heart Network, Brussels, European Society of Cardiology, Sophia Antipolis.

2. Sacco RL, Kasner SE, Broderick JP et al. An updated definition of stroke for the 21 st century. A statement for healthcare professionals from the American Heart Association/American Stroke Association. Stroke 2013; 44 (7): 2064-2089.

3. Naylor AR, Ricco JB, de Borst GJ et al. Editor's Choice - Management of Atherosclerotic Carotid and Vertebral Artery Disease: 2017 Clinical Practice Guidelines of the European Society for Vascular Surgery (ESVS). Eur J Vasc Endovasc Surg 2018; 55 (1): 3-81.

4. Rockman CB, Maldonado TS. Cerebrovascular disease: general considerations. In: Cronenwett JL, Johnston KW. Rutherford's Vascular Surgery, 8th edition. Philadelphia, Elservier Sunders; 2014. pp. 1456-1472.

5. Ozcaglayan O, K TI, Gur DO et al. Carotid arteries and vertebrobasilary system intracranial stenosis correlates with multi vessel coronary artery disease. Bratisl Med J 2019; 120 (5): 362-367.

6. AbuRahma AF, Mousa AY, Stone PA. Shunting during carotid endarterectomy. J Vasc Surg 2011; 54: 1502-1510.

7. Arnold M, Perler BA. Carotid Artery: Endarterectomy. In Cronenwett JL, Johnston KW. Rutherford's Vascular Surgery Eight Edition. Philadelphia: Elsevier Saunders; 2014. pp. 1514-1543.

8. Chongruksut W, Vaniyapong T, Rerkasem K. Routine or selective carotid artery shunting for carotid endarterectomy (and different methods of monitoring in selective shunting). Cochrane Database Syst Rev. 2014; 23 (6): CD000190. doi: 10.1002/14651858.CD000190.pub3.

9. Pedrini L, Magnoni F, Sensi L et al. Is NIRS a Reliable Method to Evaluate Clamping Ischemia during Carotid Surgery? Stroke Res Treat. Stroke Res Treat 2012; 2012: 156975. doi: 10.1155/2012/156975.

10. Ritter JC, Green D, Slim H et al. The role of cerebral oximetry in combination with awake testing in patients undergoing carotid endarterectomy under local anaesthesia. Eur J Vasc Endovasc Surg 2011; 41 (5): 599-605.

11. Ali AM, Green D, Zayed $\mathbf{H}$ et al. Cerebral monitoring in patients undergoing carotid endarterectomy using a triple assessment technique. Interact Cardiovasc Thorac Surg 2011; 12: 454-457.

12. Murkin JM, Arango M. Near-infrared spectroscopy as an index of brain and tissue oxygenation. Br J Anaesth 2009; 103: 3-13.

13. Jonsson M, Lindström D, Wanhainen A et al. Near Infrared Spectroscopy as a Predictor for Shunt Requirement During Carotid Endarterectomy. Eur J Vasc Endovasc Surg 2017; 53 (6): 783-791.
14. Ricotta JJ, Aburahma A, Ascher E et al. Society for Vascular Surgery. Updated Society for Vascular Surgery guidelines for management of extracranial carotid disease. J Vasc Surg 2011; 54 (3): 832-836.

15. GALA Trial Collaborative Group. General anaesthesia versus local anaesthesia for carotid surgery (GALA): a multicentre, randomised controlled trial. Lancet 2008; 372: 2132-2142.

16. Vaniyapong T, Chongruksut W, Rerkasem K. Local versus general anaesthesia for carotid endarterectomy. Cochrane Database Syst Rev 2013; (12): CD000126.

17. Cho JW, Jang JS. Near-Infrared Spectroscopy versus Transcranial Doppler-Based Monitoring in Carotid Endarterectomy. Korean J Thorac Cardiovasc Surg 2017; 50 (6): 448-452.

18. Li J, Shalabi A, Ji F, Meng L. Monitoring cerebral ischemia during carotid endarterectomy and stenting. J Biomed Res 2017; 31 (1): 11-16.

19. Nielsen HB. Systematic review of near-infrared spectroscopy determined cerebral oxygenation during non-cardiac surgery. Front Physiol 2014; 5: 93.

20. Samra SK, Dy EA, Welch K et al. Evaluation of a cerebral oximeter as a monitor of cerebral ischemia during carotid endarterectomy. Anesthesiology 2000; 93 (4): 964-970.

21. Wang Y, Li L, Wang T et al. The Efficacy of Near-Infrared Spectroscopy Monitoring in Carotid Endarterectomy: A Prospective, Single-Center, Observational Study. Cell Transplant 2019; 28 (2): 170-175.

22. Cao P, Giordano G, De Rango P et al. Eversion versus conventional carotid endarterectomy: late results of a prospective multicenter randomized trial. J Vasc Surg 2000; 31 (1): 19-30.

23. Antonopoulos CN, Kakisis JD, Sergentanis TN, Liapis CD. Eversion versus conventional carotid endarterectomy: a metaanalysis of randomised and non-randomised studies. Eur J Vasc Endovasc Surg 2011; 42: 751-765.

24. Roger VL. Heart disease and stroke statistics - 2012 update: a report from the American Heart Association. Circulation 2012; 125: 2-220.

25. Bennett KM, Scarborough JE, Shortell CK. Predictors of 30-day postoperative stroke or death after carotid endarterectomy using the 2012 carotid endarterectomy-targeted American College of Surgeons National Surgical Quality Improvement Program database. J Vasc Surg 2015; 61 (1): 103-111.

26. Kumas M, Altintas O, Esrefoglu M. Protective effect of ischemic preconditioning on testis injury following transient focal cerebral ischemia in diabetic rats. Bratisl Med J 2017; 118 (9): 557-563.

Received February 5, 2020. Accepted April 9, 2020. 